\title{
Sparse designs for estimating variance components of nested factors with random effects
}

\author{
R. A. Bailey ${ }^{\mathrm{a}, \mathrm{b}, *}$, Célia Fernandes ${ }^{\mathrm{c}, \mathrm{d}}$, Paulo Ramos ${ }^{\mathrm{c}, \mathrm{d}}$ \\ ${ }^{a}$ School of Mathematics and Statistics, University of St Andrews, North Haugh, \\ St Andrews, Fife, KY16 9SS, UK \\ ${ }^{b}$ School of Mathematical Sciences, Queen Mary University of London, Mile End Road, \\ London, E1 4NS, UK (emerita) \\ ${ }^{c}$ Área Departamental de Matemática, Instituto Superior de Engenharia de Lisboa, \\ Lisboa, Portugal \\ ${ }^{d}$ Centro de Matemática e Aplicações, Faculdade de Ciências e Tecnologia, Universidade \\ Nova de Lisboa, Caparica, Portugal
}

\begin{abstract}
A new class of designs is introduced for both estimating the variance components of nested factors and testing hypotheses about those variance components. These designs are flexible, and can be chosen so that the degrees of freedom are more evenly spread among the factors than they are in balanced nested designs. The variances of the estimators are smaller than those in stair nested designs of comparable size. The mean squares used in the estimation process are mutually independent, which avoids some of the problems with staggered nested designs.
\end{abstract}

Keywords: Balanced nested designs, Nested factors, Staggered nested designs, Stair nested designs, Variance components.

2020 MSC: 62J10, 62K99

\section{Introduction}

In many practical situations, measurements are taken on observational units which can be identified by the levels of a sequence $F_{1}, \ldots, F_{f}$ of factors,

\footnotetext{
${ }^{*}$ Corresponding author

Email address: rab24@st-andrews.ac.uk (R. A. Bailey)

URL: http://www-groups.mcs.st-andrews.ac.uk/ rab (R. A. Bailey)
}

Preprint submitted to Journal of Statistical Planning and Inference January 14, 2021 
each nested in the one before, and each having random effects, where $f \geq 2$. Examples include calves nested in pens nested in farms $(f=3)$, strength tests nested in preparations of material nested in boxes nested in lots $(f=4)$ in a polymerization process reported by Mason, Gunst and Hess (2003), and small leaves nested in nodes nested in branches nested in stalks nested in plants $(f=5)$, in a modification of the data reported by Trout (1985). Applications range from biometry (Searle, 1971) and agriculture to industry (Khattree, 2003).

Let $N$ be the number of observational units. The vector $\mathbf{Y}$ of observations has length $N$. We assume that the distribution of $\mathbf{Y}$ is multivariate normal, with expectation $\mathbb{E}(\mathbf{Y})=\mu \mathbf{1}_{N}$ for some unknown constant $\mu$, where $\mathbf{1}_{N}$ denotes a column vector of length $N$ with all entries equal to 1 . Denote by $\mathbf{V}$ the variance-covariance matrix of $\mathbf{Y}$.

For $j=1, \ldots, f$, it is assumed that each level of factor $F_{j}$ gives a random variable with zero mean and variance $\sigma_{j}^{2}$. Furthermore, all these random variables are independent. Let $\alpha$ and $\beta$ be two observational units (possibly with $\alpha=\beta$ ). The observations $Y_{\alpha}$ and $Y_{\beta}$ are given by $Y_{\alpha}=\mu+Z_{1 \alpha}+\cdots+Z_{f \alpha}$ and $Y_{\beta}=\mu+Z_{1 \beta}+\cdots+Z_{f \beta}$, where, for $1 \leq j \leq f, Z_{j \alpha}=Z_{j \beta}$ if $\alpha$ and $\beta$ have the same level of factor $F_{j}$ and otherwise the random variables $Z_{1 \alpha}$, $\ldots, Z_{f \alpha}, Z_{1 \beta}, \ldots, Z_{f \beta}$ are independent. Because the factors are sequentially nested, there is a largest value of $j$ such that $\alpha$ and $\beta$ have the same level of factor $F_{k}$ if and only if $k \leq j$. Then the covariance of the observations $Y_{\alpha}$ and $Y_{\beta}$ is $\sum_{k=1}^{j} \sigma_{k}^{2}$, which is zero if $j=0$. Our aim is to design an experiment to estimate the variance components $\sigma_{1}^{2}, \ldots, \sigma_{f}^{2}$, and also to test if they are non-zero. There may be little interest in the value of $\mu$.

All of our designs can be thought of as rooted trees: see Figures 1-5. The root is at depth 0 . For $j=1, \ldots, f$, each vertex at depth $j$ represents a level of factor $F_{j}$ which occurs with the level of factor $F_{j-1}$ represented by the vertex at depth $j-1$ to which it is joined. The vertices at depth $f$ are called leaves: these represent the observational units in the experiment. The pattern of entries in $\mathbf{V}$ depends on the combinatorial properties of the tree.

In addition to any limits on the value of $N$ in the experiment, there may be practical constraints on the numbers of levels of each factor. Let $m_{1}$ be the maximum number of levels of factor $F_{1}$ that it is feasible to use, and, for $j=2, \ldots, f$, let $m_{j}$ be the maximum number of levels of factor $F_{j}$ that it is feasible to use within each level of factor $F_{j-1}$. In industrial settings $m_{1}$ may be large, but there are many other practical situations in which $m_{1} \leq m_{j}$ for $2<j \leq f$. 
In Sections 2-4 we review three known families of designs for such experiments: balanced nested designs, staggered nested designs and stair nested designs. These are already in the literature: we include them here within a single approach to aid comparison between them. We show each design as a rooted tree, summarize properties of the estimators of the variance components, and comment on any difficulties in testing a hypothesis that a variance component is zero. Section 5 introduces our new designs, and develops the equivalent results for them.

Data analysis for the designs in Sections 2-5 always uses ANOVA-type sums of squares $S_{j}$ with $d_{j}$ degrees of freedom, for $j=1, \ldots, f$; the mean square $S_{j} / d_{j}$ is denoted by $M_{j}$ and the expectation of $M_{j}$ is $\gamma_{j}$, which is a known linear combination of $\sigma_{j}^{2}, \ldots, \sigma_{f}^{2}$. However, the precise meaning of $S_{j}, d_{j}, M_{j}$ and $\gamma_{j}$ is different for the different designs.

Section 6 summarizes all the designs being considered for $f=3$ and $f=4$, and compares the variances of their estimators. Finally, Section 7 gives a more wide-ranging comparison of the designs, including flexibility and degrees of freedom.

\section{Balanced nested designs}

In balanced nested designs $N$ is the product of the numbers $a_{i}$ of levels that factor $F_{i}$ has within each level of factor $F_{i-1}$. Details can be found in many standard texts, such as Cox and Solomon (2003), Sahel and Ageel (2012), Searle, Casella and McCulloch (1992) and Scheffé (1959). Here we give a brief summary, partly to establish notation and partly to enable us to compare these designs with other ones.

The first factor, $F_{1}$, has $a_{1}$ levels. Each level of $F_{1}$ nests $a_{2}$ levels of the second factor, $F_{2}$, and so on. A design with $f$ factors we write as $a_{1} / a_{2} / \cdots / a_{f}$. For $j=1, \ldots, f$, there are $c_{j}$ level combinations of the first $j$ factors, where $c_{j}=\prod_{r=1}^{j} a_{r}$, and each of these combinations nests $b_{j}$ level combinations of the following factors, where $b_{j}=c_{f} / c_{j}$. Thus $N=c_{f}=\prod_{r=1}^{f} a_{r}$. It is convenient to put $a_{0}=1, c_{0}=1$ and $b_{0}=N$. Figure 1 shows the balanced nested design $4 / 2 / 3$. The design is shown as a rooted tree, with the root at the top. In a general balanced nested design, each vertex at depth $j-1$ has $a_{j}$ branches, for $1 \leq j \leq f$.

However, in these designs the number of observations may be too large; also we are forced to divide the plots repeatedly, so there are few degrees of 
Levels of factor $F_{1}$

Levels of factor $F_{2}$

Levels of factor $F_{3}$

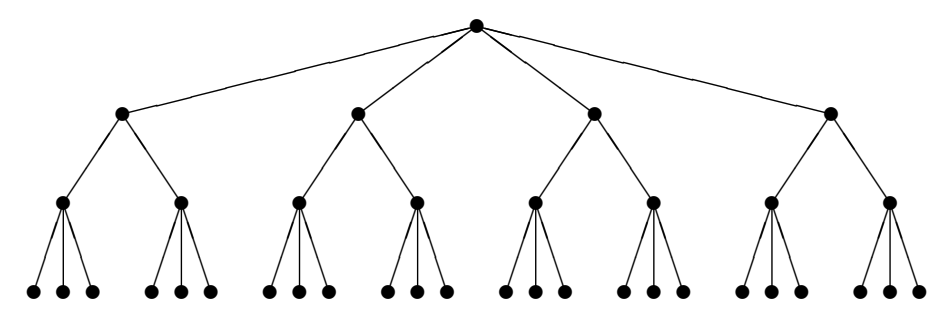

Figure 1: The balanced nested design $4 / 2 / 3$

freedom for the earlier factors and the number of degrees of freedom is not evenly distributed among the factors.

Let $\mathbf{X}_{j}$ be the $N \times c_{j}$ incidence matrix for factor $j$. Putting $\mathbf{X}_{0}=\mathbf{1}_{N}$, these matrices for a balanced nested design are

$$
\mathbf{X}_{j}=\left(\bigotimes_{r=0}^{j} \mathbf{I}_{a_{r}}\right) \otimes\left(\bigotimes_{r=j+1}^{f} \mathbf{1}_{a_{r}}\right) \quad \text { for } j=0, \ldots, f,
$$

where $\mathbf{I}_{k}$ is the identity matrix of order $k$ and $\mathbf{1}_{a_{r}}$ is the all-1 vector of length $a_{r}$. Put $\mathbf{M}_{j}=\mathbf{X}_{j} \mathbf{X}_{j}^{\prime}$ for $j=0, \ldots, f$, where $\mathbf{X}_{j}^{\prime}$ denotes the transpose of $\mathbf{X}_{j}$. Then

$$
\mathbf{M}_{j}=\left(\bigotimes_{r=0}^{j} \mathbf{I}_{a_{r}}\right) \otimes\left(\bigotimes_{r=j+1}^{f} \mathbf{J}_{a_{r}}\right) \quad \text { for } j=0, \ldots, f,
$$

where $\mathbf{J}_{k}$ is the $k \times k$ matrix with all entries equal to 1 . Then the variancecovariance matrix $\mathbf{V}$ of $\mathbf{Y}$ is given by $\mathbf{V}=\sum_{j=1}^{f} \mathbf{M}_{j} \sigma_{j}^{2}$. For $j=1, \ldots, f$, put $\mathbf{Q}_{j}=b_{j}^{-1} \mathbf{M}_{j}-b_{j-1}^{-1} \mathbf{M}_{j-1}$ and $\gamma_{j}=\sum_{r=j}^{f} b_{r} \sigma_{r}^{2}$; also put $\mathbf{Q}_{0}=b_{0}^{-1} \mathbf{M}_{0}=N^{-1} \mathbf{J}_{N}$ and $\gamma_{0}=\gamma_{1}$. Then $\mathbf{V}=\gamma_{0} \mathbf{Q}_{0}+\sum_{j=1}^{f} \gamma_{j} \mathbf{Q}_{j}$ and

$$
\mathbf{Q}_{j}=\left(\bigotimes_{r=0}^{j-1} \mathbf{I}_{a_{r}}\right) \otimes \mathbf{K}_{a_{j}} \otimes\left(\bigotimes_{r=j+1}^{f} \frac{1}{a_{r}} \mathbf{J}_{a_{r}}\right) \quad \text { for } j=1, \ldots, f,
$$

where $\mathbf{K}_{s}=\mathbf{I}_{s}-s^{-1} \mathbf{J}_{s}$. The matrices $\mathbf{Q}_{0}, \ldots, \mathbf{Q}_{f}$ are mutually orthogonal idempotents whose sum is $\mathbf{I}_{N}$, and the coefficients $\gamma_{1}, \ldots, \gamma_{f}$ are the canonical components of variance. 
For $j \geq 1$ the expectation $\mathbb{E}\left(\mathbf{Q}_{j} \mathbf{Y}\right)=\mathbf{0}_{N}$, the zero vector of length $N$. The sum of squares $S_{j}$ is defined by $S_{j}=\mathbf{Y}^{\prime} \mathbf{Q}_{j} \mathbf{Y}$. Since $\mathbf{Y}$ is multivariate normal, $S_{j} / \gamma_{j}$ has a $\chi^{2}$ distribution on $d_{j}$ degrees of freedom, where $d_{j}=$ $\operatorname{rank}\left(\mathbf{Q}_{j}\right)=c_{j}-c_{j-1}=\left(a_{j}-1\right) \prod_{r=0}^{j-1} a_{r}=\left(a_{j}-1\right) c_{j-1}$ for $j=1, \ldots, f$. Hence $\mathbb{E}\left(S_{j}\right)=d_{j} \gamma_{j}$. Moreover $S_{j}$ and $S_{k}$ are independent when $j \neq k$. For $j=1, \ldots, f$, let $M_{j}$ be the mean square $S_{j} / d_{j}$. Thus we have unbiased estimators $\hat{\gamma}_{j}=M_{j}$ for $j=1, \ldots, f$; and unbiased estimators for the variance components given by $\hat{\sigma}_{f}^{2}=\hat{\gamma}_{f}=M_{f}$ and $\hat{\sigma}_{j}^{2}=\left(M_{j}-M_{j+1}\right) / b_{j}=\left(\hat{\gamma}_{j}-\hat{\gamma}_{j+1}\right) / b_{j}$ for $j=1, \ldots, f-1$. Furthermore, $\operatorname{Var}\left(M_{j}\right)=2 \gamma_{j}^{2} / d_{j}$. Therefore $\operatorname{Var}\left(\hat{\sigma}_{f}^{2}\right)=$ $2 \gamma_{f}^{2} / d_{f}$ and $\operatorname{Var}\left(\hat{\sigma}_{j}^{2}\right)=2\left(\gamma_{j}^{2} / d_{j}+\gamma_{j+1}^{2} / d_{j+1}\right) / b_{j}^{2}$ if $j<f$.

Example 1. The balanced nested design $m / 2 / 2$ has $d_{1}=m-1, d_{2}=m$ and $d_{3}=2 m$. Also, $\hat{\sigma}_{1}^{2}=\left(M_{1}-M_{2}\right) / 4, \hat{\sigma}_{2}^{2}=\left(M_{2}-M_{3}\right) / 2$ and $\hat{\sigma}_{3}^{2}=M_{3}$. Therefore $\operatorname{Var}\left(\hat{\sigma}_{3}^{2}\right)=2 \gamma_{3}^{2} / d_{3}=\sigma_{3}^{4} / m$,

$$
\operatorname{Var}\left(\hat{\sigma}_{2}^{2}\right)=\frac{1}{4}\left(\frac{2 \gamma_{2}^{2}}{d_{2}}+\frac{2 \gamma_{3}^{2}}{d_{3}}\right)=\frac{1}{4 m}\left(8 \sigma_{2}^{4}+3 \sigma_{3}^{4}+8 \sigma_{2}^{2} \sigma_{3}^{2}\right)
$$

and

$$
\begin{aligned}
\operatorname{Var}\left(\hat{\sigma}_{1}^{2}\right)= & \frac{1}{16}\left(\frac{2 \gamma_{1}^{2}}{d_{1}}+\frac{2 \gamma_{2}^{2}}{d_{2}}\right) \\
= & \frac{1}{8 m(m-1)}\left[16 m \sigma_{1}^{4}+4(2 m-1) \sigma_{2}^{4}+(2 m-1) \sigma_{3}^{4}\right. \\
& \left.\quad+16 m \sigma_{1}^{2} \sigma_{2}^{2}+8 m \sigma_{1}^{2} \sigma_{3}^{2}+4(2 m-1) \sigma_{2}^{2} \sigma_{3}^{2}\right] .
\end{aligned}
$$

These results are summarized in Table SM.1 in the supplementary material.

Finally, if $1 \leq j<f$ and $\sigma_{j}^{2}=0$ then $M_{j} / M_{j+1}$ has an $\mathrm{F}$ distribution on $d_{j}$ and $d_{j+1}$ degrees of freedom. Thus this ratio of mean squares may be used to test the hypothesis that $\sigma_{j}^{2}=0$.

\section{Staggered and generalized staggered nested designs}

Staggered nested designs were introduced by Bainbridge (1965), and have been studied by Smith and Beverly (1981), Nelson (1983, 1995a,b), Khattree and Naik (1995), Khattree, Naik and Mason (1997), Naik and Khattree (1998) and Ojima (1998), and generalized by Ojima (2000). In such a design, factor $F_{1}$ has $n$ levels for some $n$ with $n \leq m_{1}$. Note that factors and depths 


\section{Levels of factor $F_{1}$ \\ Levels of factor $F_{2}$ \\ Levels of factor $F_{3}$}

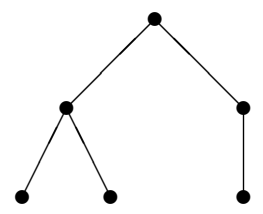

Figure 2: The unique tree $T_{3}$ for the staggered nested design with three factors

Levels of factor $F_{1}$

Levels of factor $F_{2}$

Levels of factor $F_{3}$

Levels of factor $F_{4}$

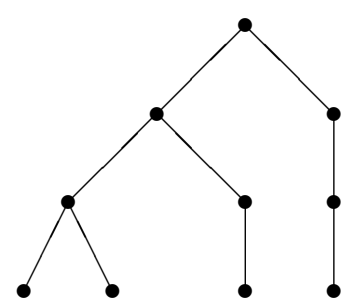

(a) $T_{4 a}$

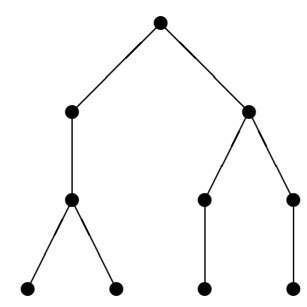

(b) $T_{4 b}$

Figure 3: The two trees for (a) staggered and (b) generalized staggered nested designs with four factors

are numbered here in the opposite order to that used by Ojima (1998), in order to be consistent with the rest of the paper.

The design has $n$ component designs, one for each level of $F_{1}$. Within each component, the design is a copy of the same rooted tree $T$, where $T$ has the property that, at each depth except the last, one vertex has two branches and the rest have one branch. Thus there are $j$ vertices at depth $j$ of $T$, for $j=1, \ldots, f$. Figure 2 shows the unique such tree for $f=3$, while the two such trees with $f=4$ are in Figure 3. We write such a design as $n / T$. When $f=2$ this is just the balanced nested design $n / 2$. In such a design, $N=n f$. Thus $N$ is constrained by $m_{1}$.

Given such a tree $T$ for $f$ factors, let $W$ be the real vector space of dimension $f$ indexed by its leaves. For $j=1, \ldots, f$, let $W_{j}$ be the subspace of $W$ consisting of vectors which are constant on each level of $F_{k}$ for $j \leq k \leq f$ and sum to zero on each level of $F_{k}$ if $1 \leq k \leq j-1$ : in particular, $W_{1}$ consists 
of the constant vectors. The condition on $T$ ensures that each of $W_{1}, \ldots, W_{f}$ is one-dimensional. For $j=1, \ldots, f$, let $\mathbf{w}_{j}$ be a vector in $W_{j}$ with length 1 ; thus $\mathbf{w}_{j}$ is unique up to multiplication by \pm 1 . It is convenient to take $\mathbf{w}_{1}$ to be $(1 / \sqrt{f}) \mathbf{1}_{f}$. For the tree $T_{3}$ in Figure 2, we may take

$$
\mathbf{w}_{2}=(1 / \sqrt{6})(1,1,-2)^{\prime} \quad \text { and } \quad \mathbf{w}_{3}=(1 / \sqrt{2})(1,-1,0)^{\prime},
$$

where the leaves are ordered from left to right as in Figure 2. For the tree $T_{4 a}$ in Figure $3(\mathrm{a})$, we may put $\mathbf{w}_{2}=(1 / \sqrt{12})(1,1,1,-3)^{\prime}, \mathbf{w}_{3}=$ $(1 / \sqrt{6})(1,1,-2,0)^{\prime}$ and $\mathbf{w}_{4}=(1 / \sqrt{2})(1,-1,0,0)^{\prime}$; while for the tree $T_{4 b}$ in Figure 3(b) we may put $\mathbf{w}_{2}=(1 / 2)(1,1,-1,-1)^{\prime}, \mathbf{w}_{3}=(1 / \sqrt{2})(0,0,1,-1)^{\prime}$ and $\mathbf{w}_{4}=(1 / \sqrt{2})(1,-1,0,0)^{\prime}$.

For $j=1, \ldots, f$, let $\mathbf{M}_{j}$ be the $f \times f$ matrix whose $(\alpha, \beta)$-entry is equal to 1 if leaves $\alpha$ and $\beta$ of $T$ have the same level of $F_{j}$; otherwise, this entry is equal to 0 . Thus $\mathbf{M}_{1}=\mathbf{J}_{f}$ and $\mathbf{M}_{f}=\mathbf{I}_{f}$, but otherwise the structure of $\mathbf{M}_{j}$ depends on the chosen tree $T$. Thus

$$
\mathbf{M}_{2}=\left[\begin{array}{lll}
1 & 1 & 0 \\
1 & 1 & 0 \\
0 & 0 & 1
\end{array}\right]
$$

for tree $T_{3}$ in Figure 2. The tree $T_{4 a}$ in Figure 3(a) has

$$
\mathbf{M}_{2}=\left[\begin{array}{llll}
1 & 1 & 1 & 0 \\
1 & 1 & 1 & 0 \\
1 & 1 & 1 & 0 \\
0 & 0 & 0 & 1
\end{array}\right] \quad \text { and } \quad \mathbf{M}_{3}=\left[\begin{array}{llll}
1 & 1 & 0 & 0 \\
1 & 1 & 0 & 0 \\
0 & 0 & 1 & 0 \\
0 & 0 & 0 & 1
\end{array}\right]
$$

while the tree $T_{4 b}$ in Figure $3(\mathrm{~b})$ has

$$
\mathbf{M}_{2}=\left[\begin{array}{llll}
1 & 1 & 0 & 0 \\
1 & 1 & 0 & 0 \\
0 & 0 & 1 & 1 \\
0 & 0 & 1 & 1
\end{array}\right] \quad \text { and } \quad \mathbf{M}_{3}=\left[\begin{array}{llll}
1 & 1 & 0 & 0 \\
1 & 1 & 0 & 0 \\
0 & 0 & 1 & 0 \\
0 & 0 & 0 & 1
\end{array}\right]
$$

Put $\mathbf{V}_{T}=\sum_{j=1}^{f} \sigma_{j}^{2} \mathbf{M}_{j}$. Then $\mathbf{V}=\mathbf{I}_{n} \otimes \mathbf{V}_{T}$, while $\mathbb{E}(\mathbf{Y})=\mu \mathbf{1}_{N}$.

For $i=1, \ldots, n$, let $\mathbf{Y}_{i}$ be the sub-vector of observations for component $i$. Put $u_{i j}=\mathbf{w}_{j}^{\prime} \mathbf{Y}_{i}$, for $1 \leq i \leq n$ and $1 \leq j \leq f$. Then $u_{i j}$ is independent of $u_{i^{\prime} k}$ if $i \neq i^{\prime}$, while the distributional properties of $u_{i j}$ do not depend on $i$, 
because the same tree $T$ is used in each component. In particular, $\mathbb{E}\left(u_{i j}\right)=0$ if $j>1$ and $\mathbb{E}\left(u_{i 1}\right)=\mu \sqrt{f}$.

Put $\phi_{j k}=\phi_{k j}=\operatorname{Cov}\left(u_{i j}, u_{i k}\right)$ for $1 \leq j \leq k \leq f$. Then $\phi_{j k}=\mathbf{w}_{j}^{\prime} \mathbf{V}_{T} \mathbf{w}_{k}=$ $\sum_{\ell=1}^{f} \mathbf{w}_{j}^{\prime} \mathbf{M}_{\ell} \mathbf{w}_{k} \sigma_{\ell}^{2}$, which is a linear combination of $\sigma_{k}^{2}, \ldots, \sigma_{f}^{2}$, because $\mathbf{M}_{\ell} \mathbf{w}_{k}=\mathbf{0}$ if $\ell<k$. In particular, put $\gamma_{j}=\phi_{j j}$ for $1 \leq j \leq f$. Then $\gamma_{j}$ is a linear combination of $\sigma_{k}^{2}$ for $j \leq k \leq f$ : the coefficients $\mathbf{w}_{j}^{\prime} \mathbf{M}_{k} \mathbf{w}_{j}$ are positive and depend on the chosen tree. Since $\mathbf{M}_{f}=\mathbf{I}_{f}$, the coefficient of $\sigma_{f}^{2}$ in $\gamma_{j}$ is always 1 , and the coefficient of $\sigma_{f}^{2}$ in $\phi_{j k}$ is zero if $j \neq k$. Ojima $(1998,2000)$ derived formulae for $\phi_{j k}$ for various trees, including those in Figures 2 and 3.

For $j \neq 1$, put $S_{j}=\sum_{i=1}^{n} u_{i j}^{2}$ and $d_{j}=n$. The variables $u_{1 j}, \ldots, u_{n j}$ are independent normal with zero mean and the same variance $\gamma_{j}$, and so $S_{j} / \gamma_{j}$ has a $\chi^{2}$ distribution on $d_{j}$ degrees of freedom. For $\gamma_{1}$, we must allow for the fact that $\mathbb{E}\left(u_{i 1}\right)=\mu \sqrt{f}$, which may be non-zero. Let $\bar{Y}$ be the overall mean of all $N$ entries in $\mathbf{Y}$, put $u_{0}=\sqrt{N} \bar{Y}, S_{1}=\sum_{i=1}^{n} u_{i 1}^{2}-u_{0}^{2}$ and $d_{1}=n-1$. Then $S_{1} / \gamma_{1}$ has a $\chi^{2}$ distribution on $d_{1}$ degrees of freedom.

For $1 \leq j \leq f$, put $M_{j}=S_{j} / d_{j}$. Now $\mathbb{E}\left(M_{j}\right)=\gamma_{j}$ and $\operatorname{Var}\left(M_{j}\right)=2 \gamma_{j}^{2} / d_{j}$ for $j=1, \ldots, f$. The ANOVA estimator $\hat{\gamma}_{j}$ for $\gamma_{j}$ is just $M_{j}$. A unique linear combination (which depends on the tree) of $\hat{\gamma}_{j}, \ldots, \hat{\gamma}_{f}$ gives the unique unbiased ANOVA estimator $\hat{\sigma}_{j}^{2}$ of $\sigma_{j}^{2}$.

An important way in which staggered nesting designs differ from the other designs in this paper is that the mean squares $M_{1}, \ldots, M_{f}$ are not all mutually independent. Since $\left(u_{i j}, u_{i k}\right)^{\prime}$ has a bivariate normal distribution, $\operatorname{Cov}\left(u_{i j}^{2}, u_{i k}^{2}\right)=2\left[\operatorname{Cov}\left(u_{i j}, u_{i k}\right)\right]^{2}=2 \phi_{j k}^{2}$, while $\operatorname{Cov}\left(u_{i j}^{2}, u_{i^{\prime} k}^{2}\right)=0$ if $i \neq i^{\prime}$. The ambiguity over the sign of $\phi_{j k}$ when $j \neq k$ does not affect this result.

Therefore, if $j$ and $k$ are both different from 1 then $\operatorname{Cov}\left(S_{j}, S_{k}\right)=2 n \phi_{j k}^{2}$ and so $\operatorname{Cov}\left(M_{j}, M_{k}\right)=2 \phi_{j k}^{2} / n$. It remains to deal with those cases in which $j=1$ or $k=1$. If $i \neq i^{\prime}$ then $u_{i j}$ is independent of $u_{i^{\prime} 1}$, and so $\operatorname{Cov}\left(u_{i j}, u_{0}\right)=\operatorname{Cov}\left(u_{i j}, u_{i 1} / \sqrt{n}\right)=\operatorname{Cov}\left(u_{i j}, u_{i 1}\right) / \sqrt{n}=\phi_{1 j} / \sqrt{n}$. Hence $\operatorname{Cov}\left(u_{i j}^{2}, u_{0}^{2}\right)=2 \phi_{1 j}^{2} / n$. If $j \neq 1$ then $\operatorname{Cov}\left(S_{1}, S_{j}\right)=2 n(1-1 / n) \phi_{1 j}^{2}=$ $2(n-1) \phi_{1 j}^{2}$, and so $\operatorname{Cov}\left(M_{1}, M_{j}\right)=2 \phi_{1 j}^{2} / n$. Finally, $u_{0}=\sum_{i=1}^{f} u_{i 1} / \sqrt{n}$ and so $\operatorname{Var}\left(u_{0}\right)=\phi_{11}$. Hence $\operatorname{Var}\left(S_{1}\right)=2 n \phi_{11}^{2}-4 n \phi_{11}^{2} / n+2 \phi_{11}^{2}=2(n-1) \phi_{11}^{2}$ and so $\operatorname{Var}\left(M_{1}\right)=2 \phi_{11}^{2} /(n-1)$.

Example 2. Using Equations (1) and (2) for the tree $T_{3}$ in Figure 2, we obtain $\gamma_{1}=\phi_{11}=\left(9 \sigma_{1}^{2}+5 \sigma_{2}^{2}+3 \sigma_{3}^{2}\right) / 3, \gamma_{2}=\phi_{22}=\left(4 \sigma_{2}^{2}+3 \sigma_{3}^{2}\right) / 3, \gamma_{3}=\phi_{33}=$ $\sigma_{3}^{2}, \phi_{12}=\sqrt{2} \sigma_{2}^{2} / 3$ and $\phi_{13}=\phi_{23}=0$. Hence $\hat{\sigma}_{3}^{2}=M_{3}, \hat{\sigma}_{2}^{2}=3\left(M_{2}-M_{3}\right) / 4$ and $\hat{\sigma}_{1}^{2}=\left(4 M_{1}-5 M_{2}+M_{3}\right) / 12$, as shown by Ojima (1998, page 792$)$. (However, note that our value of $\phi_{12}^{2}$ disagrees with his: we use our value in 
subsequent calculations.) Hence $\operatorname{Var}\left(\hat{\sigma}_{3}^{2}\right)=2 \sigma_{3}^{4} / n$,

$$
\operatorname{Var}\left(\hat{\sigma}_{2}^{2}\right)=\frac{9}{16} \times \frac{2}{n}\left(\phi_{22}^{2}+\phi_{33}^{2}\right)=\frac{1}{4 n}\left(8 \sigma_{2}^{4}+12 \sigma_{2}^{2} \sigma_{3}^{2}+9 \sigma_{3}^{4}\right)
$$

and

$$
\begin{aligned}
\operatorname{Var}\left(\hat{\sigma}_{1}^{2}\right)= & \frac{16}{144} \times \frac{2}{n-1} \phi_{11}^{2}+\frac{1}{144} \times \frac{2}{n}\left(25 \phi_{22}^{2}+\phi_{33}^{2}-40 \phi_{12}^{2}\right) \\
= & \frac{1}{2 n(n-1)}\left[4 n \sigma_{1}^{4}+\frac{20}{81}(9 n-4) \sigma_{2}^{4}+\frac{(21 n-13)}{18} \sigma_{3}^{4}\right. \\
& \left.\quad+\frac{40 n}{9} \sigma_{1}^{2} \sigma_{2}^{2}+\frac{8 n}{3} \sigma_{1}^{2} \sigma_{3}^{2}+\frac{10}{27}(9 n-5) \sigma_{2}^{2} \sigma_{3}^{2}\right] .
\end{aligned}
$$

See Table SM.2 in the supplementary material. It is the non-zero value of $\phi_{12}$ that makes these calculations more complicated than those for the designs in Sections 2, 4 and 5.

There are two impediments to performing an $\mathrm{F}$ test on the ratio $M_{j} / M_{j+1}$. One is that $M_{j}$ and $M_{j+1}$ are not independent in general. The other is that $\gamma_{j}-\gamma_{j+1}$ is not, in general, a multiple of $\sigma_{j}^{2}$ unless $j+1=f$.

\section{Stair nested designs}

Stair nested designs were introduced by Cox and Solomon (2003) and studied further by Fernandes, Ramos and Mexia (2005, 2010a,b, 2012, 2014) and Fernandes et al. (2011). They may be a good alternative to balanced nested designs since we can work with fewer observations and the amount of information for the different factors is more evenly distributed. There is effectively one component for each factor. For $j=1, \ldots, f$, factor $F_{j}$ has $a_{j}$ "active" levels in component $j$; in each other component it has only a single level if $j=1$ and otherwise a single level within each level of $F_{j-1}$. If we write + between components then the stair nested design is written as

$$
a_{1} / 1 / \cdots / 1+1 / a_{2} / 1 / \cdots / 1+\cdots+1 / \cdots / 1 / a_{f}
$$

and has $N=\sum_{r=1}^{f} a_{r}$. For $j=1, \ldots, f$, factor $F_{j}$ has $v_{j}$ levels, where $v_{j}=\sum_{r=1}^{j} a_{r}+f-j$. For example, Figure 4 shows a stair nested design with $f=3, a_{1}=3, a_{2}=2$ and $a_{3}=4$. Thus there are nine observations. 


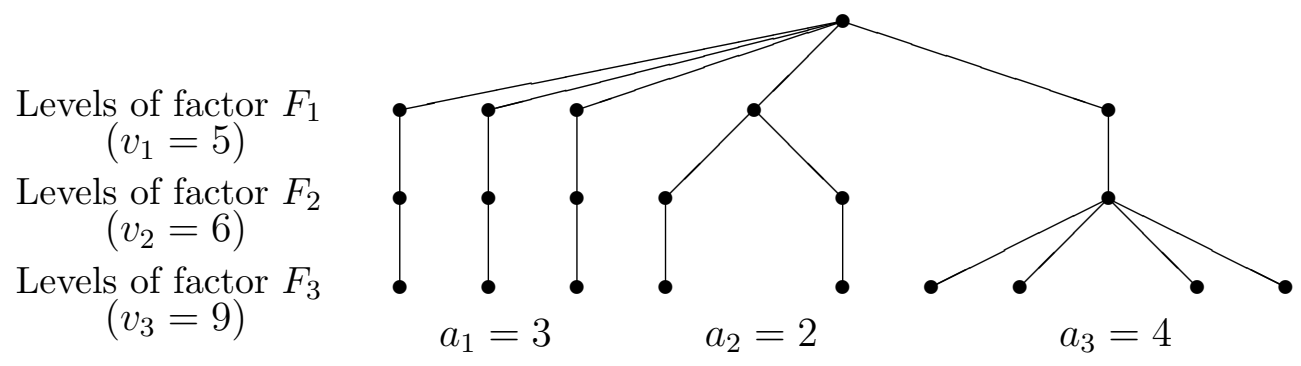

Component 1 Component 2 Component 3

Figure 4: The stair nested design $3 / 1 / 1+1 / 2 / 1+1 / 1 / 4$

Factor $F_{1}$ has $v_{1}$ levels, where $v_{1}=a_{1}+f-1$. Thus $a_{1} \leq m_{1}-f+1$. The first $a_{1}$ levels are the "active" levels for this factor; each of them is combined with a single level of each of the remaining factors. The first observation sub-vector $\mathbf{Y}_{1}$ is constituted by the observations $Y_{1}, \ldots, Y_{a_{1}}$; it has variance-covariance matrix $\mathbf{V}_{1}$ given by $\mathbf{V}_{1}=\gamma_{1} \mathbf{I}_{a_{1}}$, with $\gamma_{1}=\sum_{r=1}^{f} \sigma_{r}^{2}$.

When $1<j \leq f$, for level $a_{1}+j-1$ of the first factor we take a single level of each of the factors $F_{2}, \ldots, F_{j-1}$ and $a_{j}$ "active" levels of factor $F_{j}$. For each of these we take only one level of each of the remaining factors. The sub-vector $\mathbf{Y}_{j}$ is constituted by the observations $Y_{s_{j-1}+1}, \ldots$, $Y_{s_{j}}$, where $s_{j}=\sum_{r=1}^{j} a_{r}$. It has variance-covariance matrix $\mathbf{V}_{j}$ given by $\mathbf{V}_{j}=\omega_{j} \mathbf{J}_{a_{j}}+\gamma_{j} \mathbf{I}_{a_{j}}$, where $\omega_{j}=\sum_{r=1}^{j-1} \sigma_{r}^{2}$ and $\gamma_{j}=\sum_{r=j}^{f} \sigma_{r}^{2}$.

For $j=1, \ldots, f$, the sub-vectors $\mathbf{Y}_{j}$ are mutually independent. The sums of squares $S_{j}$ and mean squares $M_{j}$ are given by $S_{j}=\mathbf{Y}_{j}^{\prime} \mathbf{K}_{a_{j}} \mathbf{Y}_{j}$ and $M_{j}=S_{j} / d_{j}$, where $d_{j}=a_{j}-1$. Thus $S_{j}$ and $S_{k}$ are independent when $j \neq k$ and multivariate normality implies that the distribution of $S_{j} / \gamma_{j}$ is $\chi^{2}$ on $d_{j}$ degrees of freedom. Then $\mathbb{E}\left(S_{j}\right)=d_{j} \gamma_{j}$ so $\hat{\gamma}_{j}=M_{j}$ is an unbiased estimator for $\gamma_{j}$ for $j=1, \ldots, f$. Thus we have the following unbiased estimators for the variance components: $\hat{\sigma}_{f}^{2}=\hat{\gamma}_{f}=M_{f}$, and $\hat{\sigma}_{j}^{2}=M_{j}-M_{j+1}=\hat{\gamma}_{j}-\hat{\gamma}_{j+1}$ for $j=1, \ldots, f-1$. Hence $\operatorname{Var}\left(\hat{\sigma}_{f}^{2}\right)=2 \gamma_{f}^{2} / d_{f}$ and $\operatorname{Var}\left(\hat{\sigma}_{j}^{2}\right)=2\left(\gamma_{j}^{2} / d_{j}+\gamma_{j+1}^{2} / d_{j+1}\right)$ when $1 \leq j<f$.

Example 3. Consider the stair nested design with $f=3$ and $a_{1}=a_{2}=$ $a_{3}=n$. Then $d_{1}=d_{2}=d_{3}=n-1, \hat{\sigma}_{1}^{2}=M_{1}-M_{2}, \hat{\sigma}_{2}^{2}=M_{2}-M_{3}$ and 
$\sigma_{3}^{2}=M_{3}$. Therefore $\operatorname{Var}\left(\hat{\sigma}_{3}^{2}\right)=2 \gamma_{3}^{2} / d_{3}=2 \sigma_{3}^{4} /(n-1)$,

$$
\operatorname{Var}\left(\hat{\sigma}_{2}^{2}\right)=\frac{2 \gamma_{2}^{2}}{d_{2}}+\frac{2 \gamma_{3}^{2}}{d_{3}}=\frac{2}{n-1}\left(\sigma_{2}^{4}+2 \sigma_{3}^{4}+2 \sigma_{2}^{2} \sigma_{3}^{2}\right)
$$

and

$$
\begin{aligned}
\operatorname{Var}\left(\hat{\sigma}_{1}^{2}\right) & =\frac{2 \gamma_{1}^{2}}{d_{1}}+\frac{2 \gamma_{2}^{2}}{d_{2}} \\
& =\frac{2}{n-1}\left[\sigma_{1}^{4}+2 \sigma_{2}^{4}+2 \sigma_{3}^{4}+2 \sigma_{1}^{2} \sigma_{2}^{2}+2 \sigma_{1}^{2} \sigma_{3}^{2}+4 \sigma_{2}^{2} \sigma_{3}^{2}\right]
\end{aligned}
$$

See Table SM.3 in the supplementary material.

Hypothesis testing can be done exactly as in Section 2. As noted by Fernandes, Ramos and Mexia (2005), this straightforward analysis wastes $f-1$ degrees of freedom, because the mean $\mu$ is fitted separately in each component.

\section{Sparse component designs}

\subsection{The new designs}

Here we introduce a new class of designs, which we call sparse component designs. The aim is to make the degrees of freedom for estimating the variance components $\sigma_{1}^{2}, \ldots, \sigma_{f}^{2}$ nearly equal, while retaining some flexibility in the numbers of levels of each factor.

The basic idea of sparse component designs is very simple. Such a design has $u$ component designs $\Delta_{1}, \ldots, \Delta_{u}$, where $1 \leq u \leq f$. Component $\Delta_{i}$ is the balanced nested design $a_{i 1} / a_{i 2} / \ldots / a_{i f}$, where the numbers $a_{i j}$ are explained below. We write + between the components, so that $5 / 2 / 1 / 1+1 / 1 / 5 / 2$ denotes the design shown in Figure 5.

Given integers $n_{1}, \ldots, n_{f}$ with $2 \leq n_{1} \leq m_{1}-u+1$ and $2 \leq n_{j} \leq m_{j}$ for $j=2, \ldots, f$, a sparse component design is defined by a function $g$ from $\{1, \ldots, f\}$ onto $\{1, \ldots, u\}$ in the following way: for $j=1, \ldots, f$, we have $a_{i j}=1$ if $i \neq g(j)$ but $a_{g(j), j}=n_{j}$. Thus if $u=1$ then the design is a balanced nested design while if $u=f$ then the design is a stair nested design. Thus the designs in both Sections 2 and 4 are special cases of those here; we have included them as separate sections to ease the comparison with the previous literature on both. As we show in Section 5.3, component $\Delta_{g(j)}$ is the only 


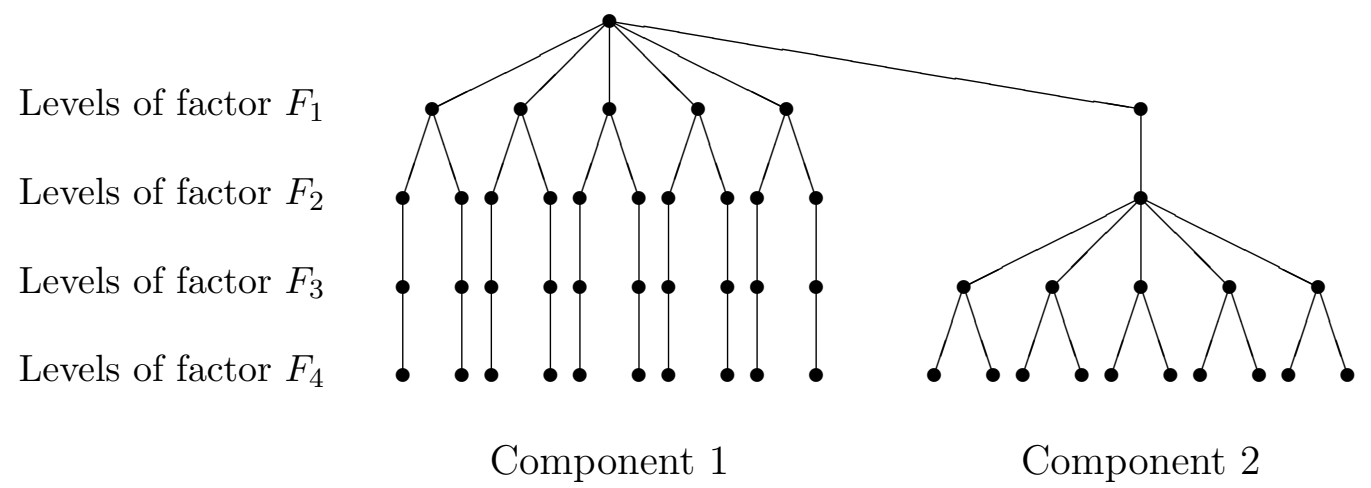

Figure 5: The design $5 / 2 / 1 / 1+1 / 1 / 5 / 2$

one which provides an estimator for the canonical component of variance $\gamma_{j}$ associated with factor $F_{j}$.

Now $N=\sum_{i=1}^{u} \prod_{j=1}^{f} a_{i j}$, which is much less than $\prod_{j=1}^{f} n_{j}$ if $u \geq 2$. Larger values of $u$ give smaller values of $N$. On the other hand, there are $u$ degrees of freedom associated with the overall mean, so there is a sense in which $u-1$ observations are wasted, so it is better not to have $u$ too large.

By changing the values of some of the $n_{j}$, we have some flexibility over the value of $N$ even if $n_{1}$ cannot be increased. To keep degrees of freedom not very different, we recommend that if $j<k$ and $g(j)=g(k)$ then $n_{j} \geq n_{k}$.

\subsection{Special sparse component designs}

In order to keep degrees of freedom nearly equal, we advocate the following special sparse component designs. In every case, the total number $N$ of observations is equal to $n f$ for a suitable choice of $n$. If $f=2$ then we recommend the balanced nested design $n / 2$, where $n \leq m_{1}$. If $f \geq 3$, our designs have $u$ components, where $u=f / 2$ if $f$ is even and $u=(f+1) / 2$ if $f$ is odd. Choose $n$ so that $2 \leq n \leq m_{1}-u+1$.

If $f$ is even then each component $\Delta_{i}$ has two values $j$ and $k$, with $1 \leq$ $j<k \leq f$, such that $a_{i j}=n, a_{i k}=2$ and $a_{i l}=1$ if $l \notin\{j, k\}$. Therefore this component has $2 n$ observations. It provides an estimator for the overall mean $\mu$; a mean square with $n-1$ degrees of freedom whose expectation is the canonical component of variance $\gamma_{j}$ associated with factor $F_{j}$; and a mean square with $n$ degrees of freedom whose expectation is the canonical component of variance $\gamma_{k}$ associated with factor $F_{k}$. These assertions are 
Table 1: The special sparse component designs for three or four factors

$$
\begin{array}{lll}
n / 2 / 1+1 / 1 / n & n / 2 / 1 / 1+1 / 1 / n / 2 \\
n / 1 / 2+1 / n / 1 & n / 1 / 2 / 1+1 / n / 1 / 2 \\
n / 1 / 1+1 / n / 2 & n / 1 / 1 / 2+1 / n / 2 / 1
\end{array}
$$

(a) Three factors

(b) Four factors

proved in Section 5.3. When $f=2$ then $u=1$ so there is a single component $\Delta_{1}$, which is the balanced nested design $n / 2$ already mentioned. Figure 5 shows one possible design with $f=4, u=2$ and $n=5$.

If $f$ is odd then there is a further component $\Delta_{i}$ for which there is a unique value of $j$ with $a_{i j}=n$, while $a_{i k}=1$ if $k \neq j$. This component has $n$ observations. It provides an estimator for $\mu$; and a mean square with $n-1$ degrees of freedom whose expectation is the canonical component of variance $\gamma_{j}$ associated with factor $F_{j}$.

It is clear that each component is balanced, and that, overall, the numbers of degrees of freedom for estimating any two canonical components of variance differ by no more than one. However, when $f \geq 3$ the total number of observations, $n f$, is much less than the number needed for balanced nesting, which is at least $n 2^{f-1}$ if the number of degrees of freedom for estimating $\sigma_{1}^{2}$ is not diminished.

When $f=3$, we obtain the three possible designs shown in Table 1(a). These have $u=2$ and $n+1 \leq m_{1}$. This table shows that our designs have some flexibility. For example, if $m_{2}<n$ but $m_{3} \geq n$ then the first design in Table 1(a) is preferred.

When $f=2 r$ and $r \geq 2$, each design is obtained from one for $2 r-1$ factors in the following way. If component $\Delta_{i}$ has $2 n$ observations then put $a_{i f}=1$; if component $\Delta_{i}$ has $n$ observations then put $a_{i f}=2$. Applying this procedure to the three designs in Table 1(a) gives the three designs in Table 1(b).

Denote by $B_{f}$ the number of special sparse component designs for $f$ factors, with the assumption that $n$ is given and $n \geq 2$. We have shown that $B_{2 r}=B_{2 r-1}$ when $r \geq 2$, that $B_{2}=1$, and that $B_{3}=B_{4}=3$.

When $f=2 r$, then each special sparse component design corresponds to 
Table 2: The 15 special sparse component designs for five factors

$$
\begin{aligned}
& n / 2 / 1 / 1 / 1+1 / 1 / n / 2 / 1+1 / 1 / 1 / 1 / n \\
& n / 2 / 1 / 1 / 1+1 / 1 / n / 1 / 2+1 / 1 / 1 / n / 1 \\
& n / 2 / 1 / 1 / 1+1 / 1 / 1 / n / 2+1 / 1 / n / 1 / 1 \\
& n / 1 / 2 / 1 / 1+1 / n / 1 / 2 / 1+1 / 1 / 1 / n \\
& n / 1 / 2 / 1 / 1+1 / n / 1 / 1 / 2+1 / 1 / 1 / n / 1 \\
& n / 1 / 2 / 1 / 1+1 / 1 / 1 / n / 2+1 / n / 1 / 1 / 1 \\
& n / 1 / 1 / 2 / 1+1 / n / 2 / 1 / 1+1 / 1 / 1 / 1 / n \\
& n / 1 / 1 / 2 / 1+1 / n / 1 / 1 / 2+1 / 1 / n / 1 / 1 \\
& n / 1 / 1 / 2 / 1+1 / 1 / n / 1 / 2+1 / n / 1 / 1 / 1 \\
& n / 1 / 1 / 1+2+1 / n / 2 / 1 / 1+1 / 1 / 1 / n / 1 \\
& n / 1 / 1 / 1+2+1 / n / 1 / 2 / 1+1 / 1 / n / 1 / 1 \\
& n / 1 / 1 / 1+1 / n+1 / n+1 / 1 / 1 \\
& n / 1 / 1 / 1 / 1+1 / n / 2 / 1 / 1+1 / 1 / n / 2 \\
& n / 1 / 1 / 1 / 1+1 / n / 1 / 2 / 1+1 / n / 1 / 2 \\
& n / 1 / 1 / 1 / 1+1 / n / 1 / 1+1 / n / 2
\end{aligned}
$$

a partition of $\{1, \ldots, 2 r\}$ into $r$ parts of size 2 . It follows that

$$
B_{2 r}=B_{2 r-1}=\frac{(2 r) !}{r ! 2^{r}} \quad \text { for } r \geq 2 .
$$

In particular, $B_{5}=B_{6}=15$ and $B_{7}=B_{8}=105$. Table 2 shows the 15 designs for five factors. Those for six factors are obtained from these. We do not anticipate that such designs will be used in practice with $f>6$.

\subsection{The algebra and the estimators}

Here we make the necessary adaptations to the results from Sections 2 and 4 to deal with sparse component designs, whether special or not. 
For $i=1, \ldots, u$ and $j=0, \ldots, f$, put $c_{i j}=\prod_{k=0}^{j} a_{i k}$ and $b_{i j}=$ $\prod_{k=j+1}^{f} a_{i k}$, where we adopt the convention that $a_{i 0}=1$ for $i=1, \ldots, u$. In particular, $c_{i f}$ is the number of observations in component $\Delta_{i}, c_{i 0}=b_{i f}=1$, and $b_{i 0}=c_{i f}$.

In component $\Delta_{i}$, put

$$
\mathbf{M}_{i j}=\left(\bigotimes_{k=0}^{j} \mathbf{I}_{a_{i k}}\right) \otimes\left(\bigotimes_{k=j+1}^{f} \mathbf{J}_{a_{i k}}\right) \quad \text { for } j=0, \ldots, f .
$$

In particular, $\mathbf{M}_{i 0}=\mathbf{J}_{c_{i f}}$. Note that, for $j=1, \ldots, f$, if $a_{i j}=1$ then $\mathbf{M}_{i j}=\mathbf{M}_{i, j-1}$ and $b_{i j}=b_{i, j-1}$, so $b_{i j}^{-1} \mathbf{M}_{i j}-b_{i, j-1}^{-1} \mathbf{M}_{i, j-1}=\mathbf{0}$, where $\mathbf{0}$ is the null matrix of appropriate size. Moreover, $b_{i j}^{-1} \mathbf{M}_{i j}$ is idempotent of rank $c_{i j}$. If $k>j$ then $\left(b_{i j}^{-1} \mathbf{M}_{i j}\right)\left(b_{i k}^{-1} \mathbf{M}_{i k}\right)=b_{i j}^{-1} \mathbf{M}_{i j}$. It follows that if $b_{i k}^{-1} \mathbf{M}_{i k}-b_{i j}^{-1} \mathbf{M}_{i j}$ is not zero then it is idempotent of rank $c_{i k}-c_{i j}$.

Now put $\mathbf{Q}_{i j}=b_{i j}^{-1} \mathbf{M}_{i j}-b_{i, j-1}^{-1} \mathbf{M}_{i, j-1}$ for $j=1, \ldots, f$. If $i \neq g(j)$ then $a_{i j}=1$ and so $\mathbf{Q}_{i j}=\mathbf{0}$. Otherwise,

$$
\mathbf{Q}_{i j}=\left(\bigotimes_{k=0}^{j-1} \mathbf{I}_{a_{i k}}\right) \otimes \mathbf{K}_{a_{i j}} \otimes\left(\bigotimes_{k=j+1}^{f} \frac{1}{a_{i k}} \mathbf{J}_{a_{i k}}\right) .
$$

Further, put $\mathbf{Q}_{i 0}=b_{i 0}^{-1} \mathbf{M}_{i 0}$. If $i=g(j)$ then $\operatorname{rank}\left(\mathbf{Q}_{i j}\right)=c_{i, j}-c_{i, j-1}=$ $c_{i, j-1}\left(a_{i j}-1\right)$. In particular, in a special sparse component design, if $a_{i j}=n$ then $\operatorname{rank}\left(\mathbf{Q}_{i j}\right)=n-1$ while if $a_{i j}=2$ then $\operatorname{rank}\left(\mathbf{Q}_{i j}\right)=n$.

Now we define some $N \times N$ block diagonal matrices. Each matrix has $u$ diagonal blocks, one for each component: the block for component $\Delta_{i}$ is a matrix of size $c_{i f} \times c_{i f}$. For $j=1, \ldots, f$, the matrix $\mathbf{M}_{j}$ has $i$-th block equal to $\mathbf{M}_{i j}$ and the matrix $\mathbf{Q}_{j}$ has $i$-th block equal to $\mathbf{Q}_{i j}$. Thus $\mathbf{Q}_{1}, \ldots, \mathbf{Q}_{f}$ are mutually orthogonal idempotents. Let $d_{j}=\operatorname{rank}\left(\mathbf{Q}_{j}\right)$ : for special sparse component designs, $d_{j}=n-1$ if $a_{g(j), j}=n$, and $d_{j}=n$ if $a_{g(j), j}=2$. For $i=1, \ldots, u$, the matrix $\mathbf{P}_{i}$ has $i$-th block equal to $\mathbf{Q}_{i 0}$ and the remaining blocks zero. Finally, $\mathbf{P}=\sum_{i=1}^{u} \mathbf{P}_{i}$ and $\mathbf{Q}=\sum_{j=1}^{f} \mathbf{Q}_{j}=\mathbf{I}_{N}-\mathbf{P}$.

By construction,

$$
\mathbf{M}_{i j}=b_{i j}\left(\sum_{k=0}^{j} \mathbf{Q}_{i k}\right)=b_{i j} \mathbf{Q}_{i 0}+b_{i j}\left(\sum_{k=1}^{j} \mathbf{Q}_{i k}\right)
$$

for $i=1, \ldots, u$ and $j=1, \ldots, f$. However, if $k \geq 1$ then $\mathbf{Q}_{i k}=\mathbf{0}$ unless 
$i=g(k)$. Hence $\mathbf{M}_{j}=\sum_{i=1}^{u} b_{i j} \mathbf{P}_{i}+\sum_{k=1}^{j} b_{g(k), j} \mathbf{Q}_{k}$. Now the variancecovariance matrix $\mathbf{V}$ of $\mathbf{Y}$ is given by

$$
\begin{aligned}
\mathbf{V}=\sum_{j=1}^{f} \sigma_{j}^{2} \mathbf{M}_{j} & =\sum_{j=1}^{f} \sum_{i=1}^{u} b_{i j} \sigma_{j}^{2} \mathbf{P}_{i}+\sum_{j=1}^{f} \sum_{k=1}^{j} b_{g(k), j} \sigma_{j}^{2} \mathbf{Q}_{k} \\
& =\sum_{i=1}^{u}\left(\sum_{j=1}^{f} b_{i j} \sigma_{j}^{2}\right) \mathbf{P}_{i}+\sum_{k=1}^{f} \gamma_{k} \mathbf{Q}_{k},
\end{aligned}
$$

with $\gamma_{k}=\sum_{j=k}^{f} b_{g(k), j} \sigma_{j}^{2}$ for $k=1, \ldots, f$.

The matrix of orthogonal projection onto the space spanned by $\mathbf{1}_{N}$ is $N^{-1} \mathbf{J}_{N}$. This does not commute with $\mathbf{V}$, so we transform the data by putting $\mathbf{Z}=\mathbf{Q Y}$. This is effectively projecting orthogonally to the grand mean within each component separately. Then $\mathbf{Q} \mathbf{1}_{N}=\mathbf{0}_{N}$, so $\mathbb{E}(\mathbf{Z})=\mathbf{0}_{N}$. Also, $\mathbf{Q P}_{i}=\mathbf{P}_{i} \mathbf{Q}=\mathbf{0}$ for $i=1, \ldots, u$, and $\mathbf{Q Q}_{j}=\mathbf{Q}_{j} \mathbf{Q}=\mathbf{Q}_{j}$ for $j=1, \ldots, f$. Hence the variance-covariance matrix of $\mathbf{Z}$ is $\mathbf{Q V Q}=\sum_{j=1}^{f} \gamma_{j} \mathbf{Q}_{j}$.

Now we can proceed almost exactly as in Section 2. For $j=1, \ldots, f$, define the sum of squares $S_{j}$ by $S_{j}=\mathbf{Z}^{\prime} \mathbf{Q}_{j} \mathbf{Z}=\mathbf{Y}^{\prime} \mathbf{Q}_{j} \mathbf{Y}$ and the mean square $M_{j}$ by $M_{j}=S_{j} / d_{j}$. These are simply the sum of squares and mean square for factor $F_{j}$ in component $\Delta_{g(j)}$. Then $\mathbb{E}\left(M_{j}\right)=\gamma_{j}$, and so $\hat{\gamma}_{j}=M_{j}$ is an unbiased estimator for $\gamma_{j}$ for $j=1, \ldots, f$. Unbiased estimators $\hat{\sigma}_{j}^{2}$ are obtained recursively. First, $\hat{\sigma}_{f}^{2}=\hat{\gamma}_{f}$, then, for $j=f-1, \ldots, 1$,

$$
\hat{\sigma}_{j}^{2}=\frac{1}{b_{g(j), j}}\left(\hat{\gamma}_{j}-\sum_{k=j+1}^{f} b_{g(j), k} \hat{\sigma}_{k}^{2}\right) .
$$

Moreover, multivariate normality implies that $S_{j}$ and $S_{k}$ are independent when $j \neq k$ and the distribution of $S_{j} / \gamma_{j}$ is $\chi^{2}$ on $d_{j}$ degrees of freedom. In general, it is not possible to give such a simple formula for $\hat{\sigma}_{j}^{2}$ as in Section 2. However, in special sparse component designs the coefficient of $\sigma_{k}^{2}$ in $\gamma_{j}$ is always either 1 or 2 if $k \geq j$.

Example 4. In the second design in Table 1(a), we have $d_{1}=d_{2}=n-1$ and $d_{3}=n$. Also, $\gamma_{1}=2 \sigma_{1}^{2}+2 \sigma_{2}^{2}+\sigma_{3}^{2}, \gamma_{2}=\sigma_{2}^{2}+\sigma_{3}^{2}$ and $\gamma_{3}=\sigma_{3}^{2}$. Hence $\hat{\sigma}_{1}^{2}=\left(M_{1}-2 M_{2}+M_{3}\right) / 2, \hat{\sigma}_{2}^{2}=M_{2}-M_{3}$ and $\hat{\sigma}_{3}^{2}=M_{3}$. Therefore $\operatorname{Var}\left(\hat{\sigma}_{3}^{2}\right)=$ $2 \gamma_{3}^{2} / d_{3}=2 \sigma_{3}^{4} / n$

$$
\operatorname{Var}\left(\hat{\sigma}_{2}^{2}\right)=\frac{2 \gamma_{2}^{2}}{d_{2}}+\frac{2 \gamma_{3}^{2}}{d_{3}}=\frac{2}{n(n-1)}\left[n \sigma_{2}^{4}+(2 n-1) \sigma_{3}^{4}+2 n \sigma_{2}^{2} \sigma_{3}^{2}\right]
$$


and

$$
\begin{aligned}
\operatorname{Var}\left(\hat{\sigma}_{1}^{2}\right)= & \frac{2}{4}\left(\frac{\gamma_{1}^{2}}{d_{1}}+\frac{4 \gamma_{2}^{2}}{d_{2}}+\frac{\gamma_{3}^{2}}{d_{3}}\right) \\
= & \frac{1}{2 n(n-1)}\left[4 n \sigma_{1}^{4}+8 n \sigma_{2}^{4}+(6 n-1) \sigma_{3}^{4}\right. \\
& \left.\quad+8 n \sigma_{1}^{2} \sigma_{2}^{2}+4 n \sigma_{1}^{2} \sigma_{3}^{2}+12 n \sigma_{2}^{2} \sigma_{3}^{2}\right]
\end{aligned}
$$

See Table SM.4 in the supplementary material.

The properties of the other two designs in Table 1(a) are summarized in Tables SM.5 and SM.6 in the supplementary material.

Hypothesis tests are not as straightforward as in Sections 2 and 4 . If $g(j)=g(j+1)$ and $\sigma_{j}^{2}=0$ then $M_{j} / M_{j+1}$ has an $\mathrm{F}$ distribution as before. If $g(j) \neq g(j+1)$ then $\gamma_{j}-\gamma_{j+1}$ is a multiple of $\sigma_{j}^{2}$ if and only if $a_{g(j), k}=$ $a_{g(j+1), k}=1$ for $k>j+1$; this is always true when $j+1=f$, and is also true for any special sparse component design in which $n_{j}=n_{j+1}=2$. In all other cases, $\gamma_{j}-\gamma_{j+1}$ is not a multiple of $\sigma_{j}^{2}$ and so an approximation like that given by Satterthwaite (1946) must be used.

\section{Comparison of designs}

\subsection{Three factors}

Tables SM.1-SM.6 in the supplementary material summarize the properties of the six types of design that we have considered for three nested factors in Sections 2-5. Table SM.1 has $N=4 m$, while $N=3 n$ in the other tables. Fair comparison of these designs requires that we put $n=4 s$ and $m=3 s$ for some integer $s$.

Table 3 summarizes the coefficients of the products of the actual variance components $\sigma_{1}^{2}, \sigma_{2}^{2}$ and $\sigma_{3}^{2}$ which occur in the formulae for the variances of their estimators in these six designs. The coefficients for the balanced nested design should be divided by $2 m(m-1)$ and those for the other designs should be divided by $2 n(n-1)$.

In every column, the largest coefficient among the first five designs occurs for the stair nested design. This shows that this design is out-performed by the staggered design and all the special sparse designs, no matter what the actual values of $\sigma_{1}^{2}, \sigma_{2}^{2}$ and $\sigma_{3}^{2}$ are. Likewise, the staggered design is always better than the sparse design $n / 1 / 2+1 / n / 1$. 
Table 3: Coefficients in the variance of the estimators of the variance components when $f=3$ : divide those in the final row by $2 m(m-1)$ and those in the other rows by $2 n(n-1)$

\begin{tabular}{c|c|c|c|c|c|c|c}
\hline \hline \multirow{2}{*}{ Type } & \multirow{2}{*}{ Design } & \multicolumn{6}{|c}{ Coefficient of } \\
& & $\sigma_{1}^{4}$ & $\sigma_{2}^{4}$ & $\sigma_{3}^{4}$ & $\sigma_{1}^{2} \sigma_{2}^{2}$ & $\sigma_{1}^{2} \sigma_{3}^{2}$ & $\sigma_{2}^{2} \sigma_{3}^{2}$ \\
\hline Sparse & $n / 2 / 1+1 / 1 / n$ & $4 n$ & $2 n-1$ & $2 n-1$ & $4 n$ & $4 n$ & $4 n-2$ \\
\hline Sparse & $n / 1 / 2+1 / n / 1$ & $4 n$ & $8 n$ & $6 n-1$ & $8 n$ & $4 n$ & $12 n$ \\
\hline Sparse & $n / 1 / 1+1 / n / 2$ & $4 n$ & $8 n$ & $6 n-1$ & $8 n$ & $8 n$ & $12 n$ \\
\hline Stair & $n / 1 / 1+1 / n / 1+1 / 1 / n$ & $4 n$ & $8 n$ & $8 n$ & $8 n$ & $8 n$ & $16 n$ \\
\hline Staggered & $n / T_{3}$ & $4 n$ & $\frac{20}{9} n-\frac{80}{81}$ & $\frac{7}{6} n-\frac{13}{18}$ & $\frac{40}{9} n$ & $\frac{8}{3} n$ & $\frac{10}{3} n-\frac{50}{27}$ \\
\hline Balanced & $m / 2 / 2$ & $4 m$ & $2 m-1$ & $\frac{1}{2} m-\frac{1}{4}$ & $4 m$ & $2 m$ & $2 m-1$ \\
\hline \hline
\end{tabular}

(a) Variance of the estimator of $\sigma_{1}^{2}$

\begin{tabular}{c|c|c|c|c}
\hline \hline \multirow{2}{*}{ Type } & \multirow{2}{*}{ Design } & \multicolumn{3}{|c}{ Coefficient of } \\
& & $\sigma_{2}^{4}$ & $\sigma_{3}^{4}$ & $\sigma_{2}^{2} \sigma_{3}^{2}$ \\
\hline Sparse & $n / 2 / 1+1 / 1 / n$ & $4 n-4$ & $8 n-4$ & $8 n-8$ \\
\hline Sparse & $n / 1 / 2+1 / n / 1$ & $4 n$ & $8 n-4$ & $8 n$ \\
\hline Sparse & $n / 1 / 1+1 / n / 2$ & $4 n$ & $2 n-1$ & $4 n$ \\
\hline Stair & $n / 1 / 1+1 / n / 1+1 / 1 / n$ & $4 n$ & $8 n$ & $8 n$ \\
\hline Staggered & $n / T_{3}$ & $4 n-4$ & $\frac{9}{2} n-\frac{9}{2}$ & $6 n-6$ \\
\hline Balanced & $m / 2 / 2$ & $4 m-4$ & $\frac{3}{2} m-\frac{3}{2}$ & $4 m-4$ \\
\hline \hline
\end{tabular}

(b) Variance of the estimator of $\sigma_{2}^{2}$

\begin{tabular}{c|c|c}
\hline \hline Type & Design & $\begin{array}{c}\text { Coefficient of } \\
\sigma_{3}^{4}\end{array}$ \\
\hline Sparse & $n / 2 / 1+1 / 1 / n$ & $4 n$ \\
\hline Sparse & $n / 1 / 2+1 / n / 1$ & $4 n-4$ \\
\hline Sparse & $n / 1 / 1+1 / n / 2$ & $4 n-4$ \\
\hline Stair & $n / 1 / 1+1 / n / 1+1 / 1 / n$ & $4 n$ \\
\hline Staggered & $n / T_{3}$ & $4 n-4$ \\
\hline Balanced & $m / 2 / 2$ & $2 m-2$ \\
\hline \hline
\end{tabular}

(c) Variance of the estimator of $\sigma_{3}^{2}$ 
The variance of the estimator of $\sigma_{3}^{2}$ is $4 \sigma_{3}^{4} / N$ for the balanced design $m / 2 / 2$, but is $6 \sigma_{3}^{4} / N$, or slightly larger, for all the other designs. This shows that the balanced design should be preferred if estimation of $\sigma_{3}^{2}$ is the primary goal.

The comparison is more complicated when we consider estimation of $\sigma_{2}^{2}$. The variance of its estimator is $\left(8 \sigma_{2}^{4}+3 \sigma_{3}^{4}+8 \sigma_{2}^{2} \sigma_{3}^{2}\right) / N$ for the balanced design; it is $\left[6 \sigma_{2}^{4}+(27 / 4) \sigma_{3}^{4}+9 \sigma_{2}^{2} \sigma_{3}^{2}\right] / N$ for the staggered design $n / T_{3}$; and it is $[6 /(N-3)]\left(\sigma_{2}^{4}+\sigma_{2}^{2} \sigma_{3}^{2}\right)+(3 / N)[(2 n-1) /(2 n-2)] \sigma_{3}^{4}$ for the sparse design $n / 1 / 1+1 / n / 2$. The staggered design beats the three remaining designs. The relative sizes of these three variances depends on the ratio $\sigma_{2}^{2} / \sigma_{3}^{2}$ and the value of $N$.

Put $x=\sigma_{2}^{2} / \sigma_{3}^{2}$. The variance for estimating $\sigma_{2}^{2}$ from $m / 2 / 2$ is less than that for $n / T_{3}$ if and only if $x<(\sqrt{31}+1) / 4 \approx 1.64$. When $N=12$ the variance from $m / 2 / 2$ is always slightly less than that from $n / 1 / 1+1 / n / 2$, but for higher values of $N$ it is less if and only if $x<[\sqrt{(n-1) /(n-4)}-1] / 2$, which rapidly approaches zero as $n$ increases. The variance from $n / T_{3}$ is less than that from $n / 1 / 1+1 / n / 2$ if and only if $x>[\sqrt{(n+5)(n-1)}+n-3] / 4$, which increases as $n$ increases.

Put $y=\sigma_{1}^{2} / \sigma_{3}^{2}$. A similar investigation shows that the design which estimates $\sigma_{1}^{2}$ with the smallest variance is one of the balanced design, the staggered design, and the sparse design $n / 2 / 1+1 / 1 / n$. Any of these can be best, depending on the value of $N$ and quadratic inequalities involving $x$ and $y$.

\subsection{Four factors}

Calculations similar to those in Examples 1-4 give full details for the designs for four nested factors discussed in Sections 2-5. The results are shown in Tables SM.7-SM.13 in the supplementary material. Now $N=8 \mathrm{~m}$ in Table SM.7 and $N=4 n$ in Tables SM.8-SM.13, so fair comparison of these designs needs $n=2 m$.

Table 4 is the analogue of Table 3. Again, the coefficients for the balanced nested design should be divided by $2 m(m-1)$ and those for the other designs should be divided by $2 n(n-1)$.

Once again, the stair nested design gives larger variances than all the other designs, except possibly for the estimators of $\sigma_{1}^{2}$ and $\sigma_{2}^{2}$ in the balanced design. For estimating $\sigma_{4}^{2}$, the variance of the estimator from the balanced design $m / 2 / 2 / 2$ is one half (or less) of the variance from any other listed

design. For estimating $\sigma_{3}^{2}$, the variance from the balanced design $m / 2 / 2 / 2$ is 


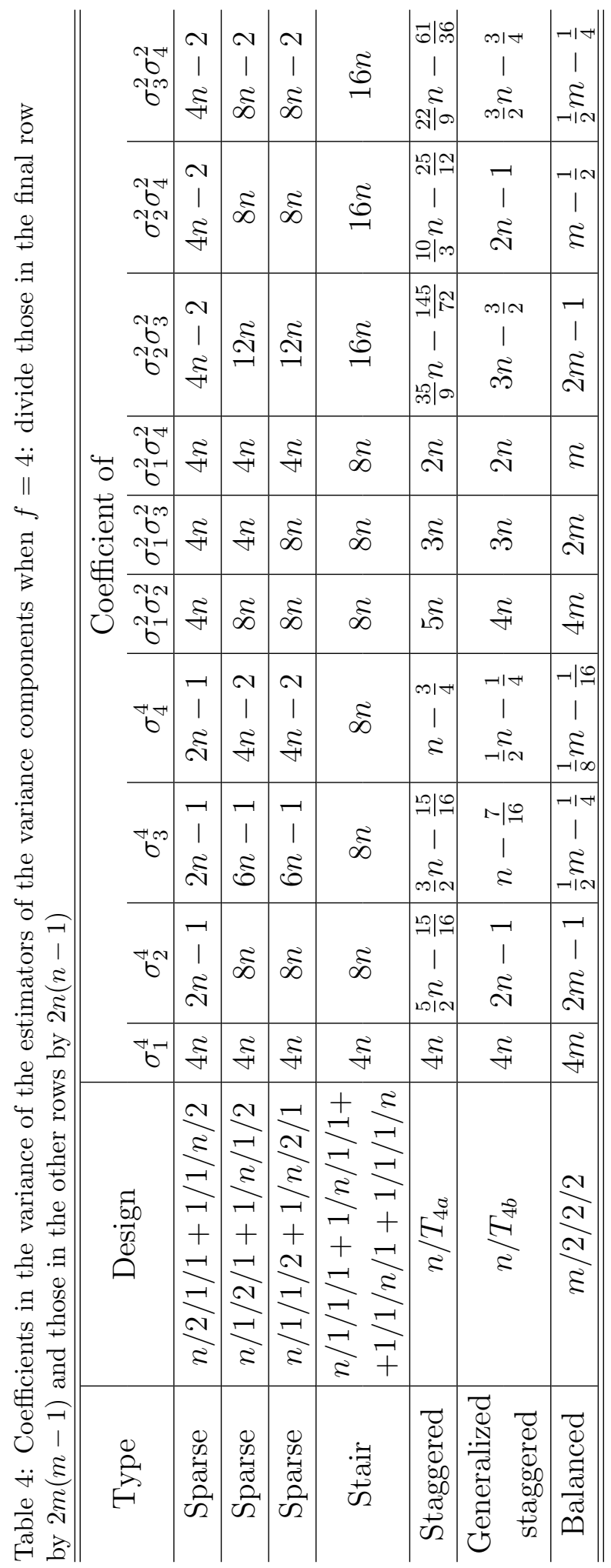

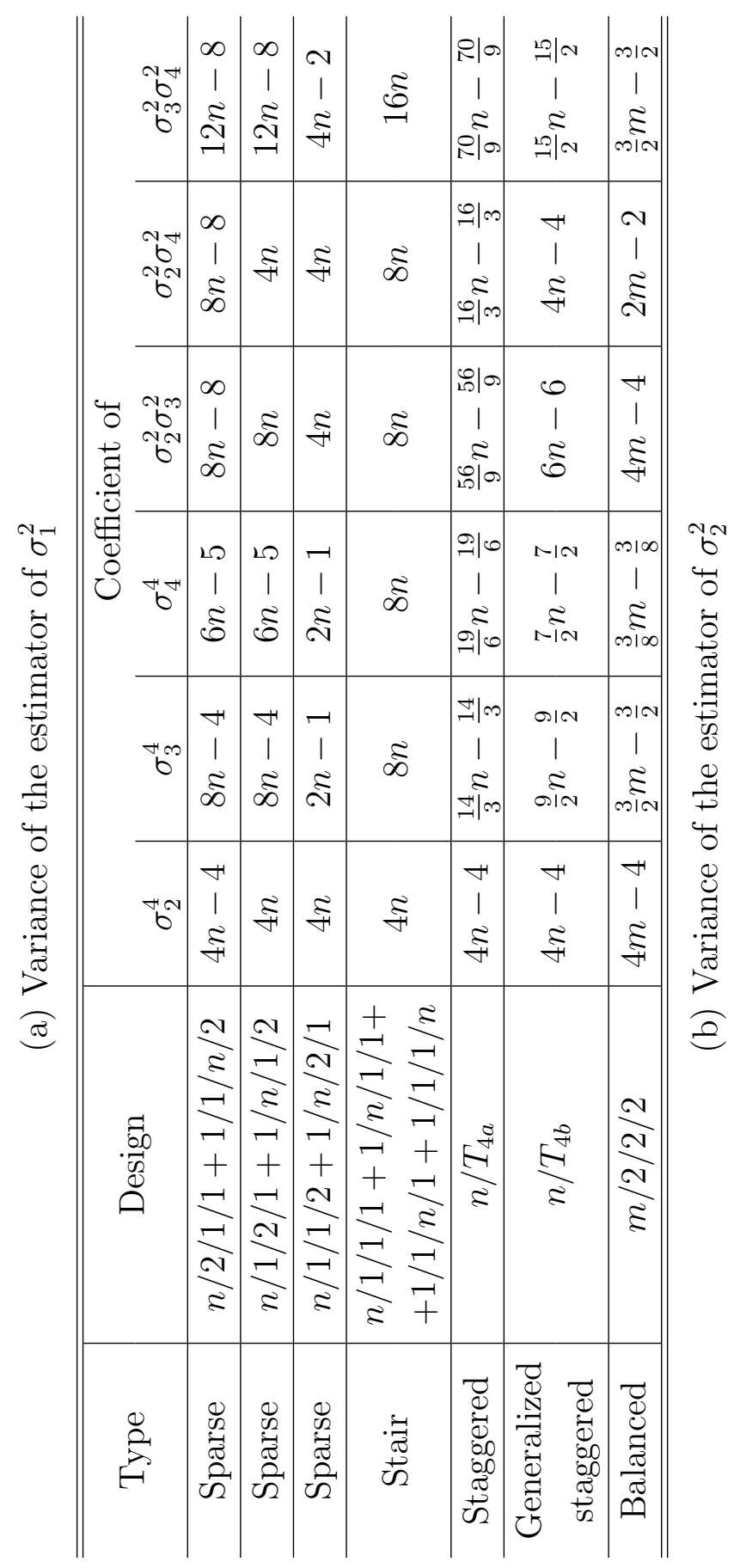


Table 4: Coefficients in the variance of the estimators of the variance components when $f=4$ : divide those in the final row by $2 m(m-1)$ and those in the other rows by $2 n(n-1)$

\begin{tabular}{c|c|c|c|c}
\hline \hline \multirow{2}{*}{ Type } & Design & \multicolumn{3}{|c}{ Coefficient of } \\
& & $\sigma_{3}^{4}$ & $\sigma_{4}^{4}$ & $\sigma_{3}^{2} \sigma_{4}^{2}$ \\
\hline Sparse & $n / 2 / 1 / 1+1 / 1 / n / 2$ & $4 n$ & $2 n-1$ & $4 n$ \\
\hline Sparse & $n / 1 / 2 / 1+1 / n / 1 / 2$ & $4 n-4$ & $8 n-8$ & $8 n-8$ \\
\hline Sparse & $n / 1 / 1 / 2+1 / n / 2 / 1$ & $4 n-4$ & $8 n-8$ & $8 n-8$ \\
\hline Stair & $n / 1 / 1 / 1+1 / n / 1 / 1+$ & $4 n$ & $8 n$ & $8 n$ \\
\hline Staggered & $+1 / 1 / n / 1+1 / 1 / 1 / n$ & & & \\
\hline Generalized & $n / T_{4 a}$ & $4 n-4$ & $\frac{9}{2} n-\frac{9}{2}$ & $6 n-6$ \\
staggered & $n / T_{4 b}$ & $4 n-4$ & $8 n-8$ & $8 n-8$ \\
\hline Balanced & $m / 2 / 2 / 2$ & $2 m-2$ & $\frac{3}{4} m-\frac{3}{4}$ & $2 m-2$ \\
\hline \hline
\end{tabular}

(c) Variance of the estimator of $\sigma_{3}^{2}$

\begin{tabular}{c|c|c}
\hline \hline Type & Design & $\begin{array}{c}\text { Coefficient of } \\
\sigma_{4}^{4}\end{array}$ \\
\hline Sparse & $n / 2 / 1 / 1+1 / 1 / n / 2$ & $4 n-4$ \\
\hline Sparse & $n / 1 / 2 / 1+1 / n / 1 / 2$ & $4 n-4$ \\
\hline Sparse & $n / 1 / 1 / 2+1 / n / 2 / 1$ & $4 n-4$ \\
\hline Stair & $\begin{array}{c}n / 1 / 1 / 1+1 / n / 1 / 1+ \\
+1 / 1 / n / 1+1 / 1 / 1 / n\end{array}$ & $4 n$ \\
\hline Staggered & $n / T_{4 a}$ & $4 n-4$ \\
\hline Generalized & $n / T_{4 b}$ & $4 n-4$ \\
staggered & $m / 2 / 2 / 2$ & $m-1$ \\
\hline Balanced &
\end{tabular}

(d) Variance of the estimator of $\sigma_{4}^{2}$ 
again the smallest, but those from the staggered design $n / T_{4 a}$ and the sparse design $n / 2 / 1 / 1+1 / 1 / n / 2$ are not much larger.

If $\sigma_{2}^{2}$ is much smaller than $\sigma_{3}^{2}$ and $\sigma_{4}^{2}$ then the smallest variances of the estimator of $\sigma_{2}^{2}$ come from the balanced design $m / 2 / 2 / 2$ and the sparse design $n / 1 / 1 / 2+1 / n / 2 / 1$. If $\sigma_{2}^{2}$ is much bigger than $\sigma_{3}^{2}$ and $\sigma_{4}^{2}$ then the sparse design $n / 1 / 1 / 2+1 / n / 2 / 1$ and the generalized staggered design $n / T_{4 b}$ give the smallest variances of the estimator of $\sigma_{2}^{2}$.

In Table 4(a), the coefficients for the generalized staggered design $n / T_{4 b}$ are the smallest in every column except that for $\sigma_{4}^{2}$, and that for $\sigma_{3}^{2} \sigma_{4}^{2}$ if $n \geq 4$. Thus this design gives the smallest variance of the estimator of $\sigma_{1}^{2}$ unless $\sigma_{4}^{2}$ is much bigger than all of $\sigma_{1}^{2}, \sigma_{2}^{2}$ and $\sigma_{3}^{2}$, in which case the balanced design $m / 2 / 2 / 2$ might be preferred.

\subsection{Choice of design}

If the aim of the experiment is estimation of the variance components $\sigma_{1}^{2}, \ldots, \sigma_{f}^{2}$ then we seek unbiased estimators with low variance. All of the designs discussed here give unbiased estimators, but the relative magnitudes of the variances of those estimators can change, both as the number $N$ of observations changes and according to the relative sizes of $\sigma_{1}^{2}, \ldots, \sigma_{f}^{2}$. In planning such an experiment, we recommend using the actual number $N$ together with any prior knowledge about the relative sizes of $\sigma_{1}^{2}, \ldots, \sigma_{f}^{2}$ to compare the likely variances of the estimators. The experimenter can decide whether to try to minimize $\sum_{i=1}^{f} \operatorname{Var}\left(\hat{\sigma}_{i}^{2}\right)$ or to give some variances higher weights.

If the aim of the experiment is to test the null hypotheses $\mathrm{H}_{0 i}: \sigma_{i}^{2}=0$ for $i=1, \ldots, f-1$, or to give confidence intervals for the values of $\sigma_{1}^{2}$, $\ldots, \sigma_{f}^{2}$, then degrees of freedom play a double role, as they affect power and length of confidence interval both through the sizes of the variances and through the distributions of the test statistics. Now lack of independence between the mean squares for the staggered and generalized staggered nested designs makes these designs problematic, while the very uneven distribution of degrees of freedom in the balanced nested designs makes them inferior to other designs with the same variances.

Of course, for any of the designs in Sections 2-5, it is perfectly possible that $M_{j}<M_{j+1}$ for some $j$ with $j<f$. One way that this can occur is that $\mathbf{V}$ has the form specified in the relevant section but there is negative correlation between observations with the same level of $F_{j}$. This can happen if there is competition for resources within each level of $F_{j}$. For example, if 
the design in Figure 1 is used for plants nested in trays nested in greenhouses then $\gamma_{2}$ could be less than $\gamma_{3}$. See Nelder (1954) and Bailey and Brien (2016).

Even if there is no such negative correlation, the variability of both $M_{j}$ and $M_{j+1}$ can give an outcome with $M_{j}<M_{j+1}$ when $\sigma_{j}^{2}>0$. The smaller is $d_{j}$, the more likely is this to occur. In such cases, some people recommend concluding that $\sigma_{j}^{2}=0$ and pooling $S_{j}$ and $S_{j+1}$, thus using $\left(S_{j}+S_{j+1}\right) /\left(d_{j}+\right.$ $\left.d_{j+1}\right)$ as the estimator for $\gamma_{j+1}$. However, because this is done only when $M_{j}<M_{j+1}$, this procedure introduces bias, as shown by Wolde-Tsadik and Afifi (1980); Draper and Smith (1998); Janky (2000); Gilmour and Trinca (2012). We do not recommend such pooling. The designs recommended in Sections $3-5$ all have $N / f-1 \leq d_{j} \leq N / f$ for $1 \leq j \leq f$. The balanced nested designs $m / 2 / 2$ have $d_{2}=N / 4=d_{1}+1$ and the balanced nested designs $m / 2 / 2 / 2$ have $d_{2}=N / 8=d_{1}+1$. Hence this problem is more likely to occur if a balanced nested design is used.

\section{Discussion}

Unlike staggered nested designs, sparse component designs retain the simplicity and independence of classical orthogonal designs. If there is more than one component, the distribution of degrees of freedom can be made more even than in balanced nested designs. For given constraints on the numbers of levels and number of observations, there is more flexibility in the choice of sparse component designs than there is with balanced nested designs, staggered nested designs or stair nested designs. A small number of degrees of freedom are wasted in sparse component designs, but this number is less than the number wasted in stair nested designs unless the number of components is maximal.

For the polymerization example in Section 1, Mason, Gunst and Hess (2003) proposed the staggered nested design 30/T $T_{4 a}$. This design has 120 observations, and gives 29, 30, 30, 30 degrees of freedom for lots, boxes, preparations and tests respectively. As shown in Section 6, if either $\sigma_{1}^{2}$ or $\sigma_{2}^{2}$ is large then the generalized staggered design $30 / T_{4 b}$ will give smaller variances for the same number of observations and degrees of freedom.

It appears from Mason, Gunst and Hess (2003) that $m_{1} \geq 30$ and $m_{2}=$ 100. If $m_{3}$ and $m_{4}$ are both small then either of the sparse designs $30 / 1 / 1 / 2+$ $1 / 30 / 2 / 1$ and $30 / 1 / 2 / 1+1 / 30 / 1 / 2$ can be used, giving 29 or 30 degrees of freedom for each factor. With these numbers, the wasting of a single degree 
of freedom has no practical consequence. Inference from these sparse designs is more straightforward than from the designs $30 / T_{4 a}$ and $30 / T_{4 b}$.

The plant example in Section 1 has $f=5$. The original experiment reported by Trout (1985) had $f=4$, using a single plant. It seems that the physical constraints were $m_{1}=5, m_{2}=2, m_{3}=5$ and $m_{4}=3$. The original design was the balanced nested design $5 / 2 / 5 / 3$, which has 150 observations. It has 4, 5, 40 and 100 degrees of freedom for stalks, branches, nodes and leaves respectively. If more plants are available, an alternative balanced nested design is $9 / 2 / 2 / 2 / 2$, using 9 plants and 144 observations. This has 8 , $9,18,36$ and 72 degrees of freedom for plants, stalks, branches, nodes and leaves respectively. Even if there is no interest in the variance component for plants, this is an improvement on the original design.

Because $m_{1}=5$ and $m_{2}=2$, the only possible special sparse component design for a single plant is $4 / 2 / 1 / 1+1 / 1 / 4 / 2$, with 16 observations and 3, 4, 3 and 4 degrees of freedom. However, the second component may be replaced by $1 / 1 / 5 / 2$ or $1 / 1 / 4 / 3$ or $1 / 1 / 5 / 3$, giving designs with 18,20 or 23 observations and increased degrees of freedom for nodes and leaves. The largest possible staggered nested design with a single plant is $5 / T_{4 a}$ or $5 / T_{4 b}$, both of which have 20 observations and 4, 5, 5 and 5 degrees of freedom.

If more plants are available, there are several other designs with $f=5$ and $16<N<144$. These can be useful even if there is no interest in the variance component for plants. One possibility is $2 / 5 / 2 / 1 / 1+2 / 1 / 1 / 5 / 2$, which uses four plants and has 40 observations. For the factor plants, this does not satisfy the condition about the function $g$ given in Section 5.1. In any case, it has too few degrees of freedom for any meaningful estimate of the plants variance component. However, the 36-dimensional space orthogonal to plants satisfies the conditions in Section 5.3, and gives independent estimators of the remaining variance components with 8, 10, 8 and 10 degrees of freedom.

This example shows why the condition about $g$ in Section 5.1 is useful in general. If there is a factor $F_{j}$ and different components $\Delta_{i}$ and $\Delta_{h}$ in which $a_{i j}>1$ and $a_{h j}>1$ then both of these components provide estimators for $\sigma_{j}^{2}$. Combining information from these two estimators is not straightforward in general. This is essentially the same problem as occurs for the balanced design for rectangular blocks if it is assumed that there are variance components for rows, columns and plots but not for blocks: see Bailey, Ferreira, Ferreira and Nunes (2016). Nevertheless, it may be sensible to relax the condition about $g$ if no suitable design can otherwise be found.

Overall, sparse component designs appear more flexible than balanced 
nested designs, stair nested designs and staggered nested designs. They can be constructed with many fewer observations than balanced nested designs. Since the implementation cost is often a decisive factor in practical experiments, these new designs will be a strong alternative to the balanced nested designs. They can give a good distribution of degrees of freedom among the variance components. Inference is more straightforward than for staggered nested designs. Thus we have no hesitation in recommending them for experiments with hierarchically nested factors when they are designed either to estimate the magnitudes of variance components or to test the hypotheses that these are non-zero.

Acknowledgements. This work is funded by National Funds through the FCT - Fundação para a Ciência e a Tecnologia, I.P., under the scope of the project UIDB/00297/2020 (Center for Mathematics and Applications).

Appendix: Supplementary Material. Supplementary material related to this article can be found online at https://(add the address here).

\section{References}

Bailey, R. A., Brien, C. J., 2016. Randomization-based models for multitiered experiments. I. A chain of randomizations. Annals of Statistics 44, 11311164.

Bailey, R. A., Ferreira, S. S., Ferreira, D., Nunes, C., 2016. Estimability of variance components when all model matrices commute. Linear Algebra and its Applications 492, 144-160.

Bainbridge, T. R., 1965. Staggered nested designs for estimating variance components. Indust. Quality Control 22, 12-20.

Cox, D. R., Solomon, P. J., 2003. Components of Variance, Monographs on Statistics and Applied Probability, vol. 97. Chapman \& Hall/CRC, Boca Raton.

Draper, N. R., Smith, H., 1998. Applied Regression Analysis, 3rd ed. Wiley, New York.

Fernandes, C., Ramos, P., Mexia, J., 2005. Optimization of nested step designs. Biometrical Letters 42, 143-149. 
Fernandes, C., Ramos, P., Mexia, J., 2010a. Balanced and step nesting designs - Application for cladophylls of asparagus. JP Journal of Biostatistics 4, 279-287.

Fernandes, C., Ramos, P., Mexia, J., 2010b. Algebraic structure of step nesting designs. Discussiones Mathematicae: Probability and Statistics 30, $221-235$.

Fernandes, C., Ramos, P., Mexia, J., 2012. Crossing balanced and stair nested designs. Electronic J. Linear Algebra 25, 22-47.

Fernandes, C., Ramos, P., Mexia, J., 2014. Algebraic structure for the crossing of balanced and stair nested designs. Discussiones Mathematicae: Probability and Statistics 34, 71-88.

Fernandes, C., Ramos, P., Mexia, J., Carvalho, F., 2011. Models with stair nesting. AIP Conference Proceedings 1389, 1627-1630.

Gilmour, S. G., Trinca, L. A., 2012. Optimum design of experiments for statistical inference (with discussion). J. Royal Statistical Society: Series C (Applied Statistics) 61, 345-401.

Janky, D. G., 2000. Sometimes pooling for analysis of variance hypothesis tests: A review and study of a split-plot model. The American Statistician 54, 269-279.

Khattree, R., 2003. Subsampling designs in industry: statistical inference for variance components. Chapter 21 in: Khattree, R. and Rao, C. R. (Eds), Handbook of Statistics, Volume 22. Elsevier, pp. 765-794.

Khattree, R., Naik, D. N., 1995. Statistical tests for random effects in staggered nested designs. J. Applied Statistics 22, 495-505.

Khattree, R., Naik, D. N., Mason, R. L., 1997. Estimation of variance components in staggered nested designs. J. Applied Statistics 24, 395-408.

Mason, R. L., Gunst, R. F., Hess, J. L., 2003. Statistical Design and Analysis of Experiments: With Applications to Engineering and Science, 2nd ed. John Wiley \& Sons, Hoboken, New Jersey. 
Naik, D. N., Khattree, R., 1998. A computer program to estimate variance components in staggered nested designs. J. Quality Technology 30, 292297.

Nelder, J. A., 1954. The interpretation of negative components of variance. Biometrika 41, 544-548.

Nelson, L. S., 1983. Variance estimation using staggered nested designs. J. Quality Technology 15, 195-198.

Nelson, L. S., 1995a. Using nested designs: I. Estimation of standard deviations. J. Quality Technology 27, 169-171.

Nelson, L. S., 1995b. Using nested designs: II. Confidence limits for standard deviations. J. Quality Technology 27, 265-267.

Ojima, Y., 1998. General formulae for expectations, variances and covariances of the mean squares for staggered nested designs. J. Applied Statistics 25, 785-799.

Ojima, Y., 2000. Generalized staggered nested designs for variance components estimation. J. Applied Statistics 27, 541-553.

Sahel, H., Ageel, M. I., 2012. The Analysis of Variance. Fixed, Random and Mixed Models. Springer, New York.

Satterthwaite, F. E., 1946. An approximate distribution of estimates of variance components. Biometrics Bulletin 2, 110-114.

Scheffé, H., 1959. The Analysis of Variance. John Wiley \& Sons, Inc., New York.

Searle, S. R., 1971. Topics in variance component estimation. Biometrics 27, 1-76.

Searle, S. R, Casella, G., McCulloch, C. E., 1992. Variance Components. Wiley, New York.

Smith, J. R., Beverly, J. M., 1981. The use and analysis of staggered nested factorial designs. J. Quality Technology 13, 166-173. 
Trout, J. R., 1985. Design and analysis of experiments to estimate components of variance: two case studies. In: Snee, R. D., Hare, L. B., Trout, J. R. (Eds.), Experiments in Industry: Design, Analysis and Interpretation of Results. American Society for Quality Control, Milwaukee, pp. $75-88$.

Wolde-Tsadik, G., Afifi, A. A., 1980. A comparison of the "sometimes pool", "sometimes switch" and "never pool" procedures in the two-way ANOVA random effects model. Technometrics 22, 367-373. 